\title{
The glial actin cytoskeleton regulates neuronal ciliogenesis
}

Cell Research (2017) 27:448-451. doi:10.1038/cr.2016.131; published online 11 November 2017

\section{Dear Editor,}

Cilia are remarkable microtubule (MT)-based organelles that are essential for cell motility, sensory perception and signal transduction [1]. Ciliary defects have been implicated in various human diseases [1]. Although the rod-shaped cilium morphology and the axonemal structure characterized by nine-doublet MTs were described decades ago $[2,3]$, the molecular regulations of cilium morphology and axonemal structure are still poorly defined. The formation and maintenance of cilia require bidirectional intraflagellar transport (IFT) that is powered by MT-based kinesin-2 and IFT-dynein motor proteins [1, 4]. Despite important progresses in identifying ciliary components and delineating the regulatory events of IFT [1, 4], it remains elusive how other cytoskeletal elements such as actin contribute to cilium assembly and how environmental cues direct ciliogenesis in metazoans. Emerging evidence shows that actomyosin facilitates the recruitment of IFT materials to basal bodies in Chlamydomonas [5]. In contrast, actin-based membrane trafficking was postulated to inhibit cilium formation by removing ciliary precursors from basal bodies in mammalian cell cultures $[6,7]$, raising the question of whether ciliogenesis in different cell types may rely on distinct actin contributions. Importantly, little is known about the role of actin in ciliogenesis in animals.

The C. elegans chemosensory organs, the amphids and phasmids, comprise ciliated dendrites of sensory neurons plus two ensheathing glial cells called socket and sheath cells, which form a cylindrical channel surrounding the sensory cilia (Figure 1A-1B and Supplementary information, Figure S1A) [3]. To identify the potential functions of the actin cytoskeleton in the formation of sensory cilia in $C$. elegans neurons, we determined the dye-filling (Dyf) phenotype of C. elegans mutants with defective actin regulators. Previous studies have established that the inability of sensory neurons to take up the dye DiI (Dyf defect) through the openings of chemosensory organs correlates with abnormalities in the ciliary structure or the sensory compartments [3]. Through this analysis, we uncovered that an actin nucleation-promoting factor Wiskott-Aldrich syndrome protein (WASP) homologue, WSP-1A, is involved in cilium formation in C. elegans (Supplementary information, Figure S1B). While a previous study showed that the wsp-1a (gm324) mutation reversed the enlargement of sensory channel in a Patched-related gene daf- 6 mutant, this study did not detect the Dyf defect in the wsp-la (gm324) single mutant and suggested that WSP-1A is not required for ciliogenesis in C. elegans [8]. However, cilium structure and IFT have not been examined closely. We confirmed that the amphid sensory neurons of wsp-1a (gm324) mutants indeed took up DiI. However, 55\% of the phasmid neurons failed to take up DiI ( $n=390$; Supplementary information, Figure S1B). The discrepancy between the amphids and phasmids likely resulted from incomplete penetration of the Dyf defect of wsp-la (gm324) mutants, and the Dyf defect is usually scored if none of the eight amphid neurons or none of the two phasmid neurons are filled with DiI. To better assess the cilium structure and IFT in wsp-la mutants, we genetically introduced these animals with OSM-6/IFT52::mCherry to label the fulllength cilium. The animals lacking WASP function either lost their ciliary distal segments or formed curved cilia, and IFT was only occasionally detected by kymographs in the remaining ciliary segments (Figure $1 \mathrm{C}-1 \mathrm{E}$ ).

Next, we performed serial-section transmission electron microscopy (ss-TEM) and focused ion beam scanning electron microscopy (FIB-SEM) of the sensory organs in wsp-1a (gm324) mutant animals. While ss-TEM cross sections of the wild-type (WT) amphid sensory organ showed ten channel cilia, the sensory compartment of wsp-1a mutants contains fewer cilia and the amphid channel is filled with highly electron-dense material,which was not detected in WT sensory channels (Figure 1F, Supplementary information, Figure S2B and Movie S1-S2). Intriguingly, 3D tomographic reconstructions of FIB-SEM images from cross sections revealed that the distal segments of several cilia split into two portions, generating Y-shaped morphology (Figure $1 \mathrm{G}$ and $1 \mathrm{~K}$ ). We confirmed these phenotypes in wsp-la mutants by reconstructing FIB-SEM images from longitudinal sections of the amphid (Figure 1I and Supplementary information, Figure S2A). Furthermore, we performed TEM to compare the axonemal structures in Y-shaped cilia with those 
in normal cilia. A neuronal cilium in sensory channel normally extends longitudinally into the middle and distal segments that consists of 9 doublet and 9 singlet outer MTs, respectively [2, 3] (Figure 1L-1M and Supplementary information, Figure S1C). In the middle segments of wsp-1a mutant cilia, fewer doublet MTs could be found at the axonemal periphery and doublets appeared at the axonemal center, whereas the distal segments showed abnormal presence of doublets and reduced number of singlet MTs (Figure 1L-1M and Supplementary information, Figure S1C). Our data indicate that WASP is involved in the regulation of cilium morphology and the organization of MTs at the axonemal periphery.

To understand how WASP contributes to ciliogenesis, we generated GFP knock-in (KI) animals to accurately determine the in vivo expression and localization of endogenous WSP-1A. GFP::WSP-1A is specifically localized in the socket cells but is barely detectable in the cilia or the sheath cells (Figure $1 \mathrm{~N}$ ). We carried out cell type-specific genetic rescue experiments to determine in which cells WSP-1A functions during ciliogenesis. In agreement with the specific localization of GFP::WSP-1A in the socket cells, the Dyf defect in wsp$1 a(g m 324)$ worms could be rescued by expression of wsp- $1 a$ driven by the socket cell-specific promoter (Pitr1 ), and the cilium morphology in the rescued worms was indistinguishable from that of WT animals (Figure 1P and Supplementary information, Figure S2E-S2F). However, the expression of $w s p-1 a$ driven by the ciliated neuron-specific promoter $(\mathrm{P} d y f-1)$ failed to rescue the Dyf defector abnormality in cilium morphology (Figure 1P and Supplementary information, Figure S2E-S2F). These genetic rescue data provided strong evidence that wsp- $1 a$ functions in the glial socket cells but not in sensory neurons to promote neuronal cilium formation. Consistently, the amphid channel at the distal tip of the socket cell collapsed in $w s p-1 a$ mutant animals (Figure $1 \mathrm{~F}$ and $1 \mathrm{H}$ ). Our FIB-SEM and 3D reconstructions support the TEM observations that lack of WASP function reduced the size of the socket channel (Figure 1G, 1J and Supplementary information, Movies S3-S5). Thus, WASP contributes to neuronal ciliogenesis by regulating the appropriate size of the sensory neuron channel.

We next investigated the WASP downstream components including the Arp2/3 complex and the actin cytoskeleton in sensory organs. Using ARX-2::GFP (an Arp2 homolog in C. elegans) knock-in animals, we showed that ARX-2::GFP fluorescence is specifically localized in the socket cells but is not detected in the cilia or the sheath cells (Figure 1O), which is identical to the localization pattern of GFP::WSP-1A. By using the cell type-specific expression of GFP-tagged calponin homology domain of the F-actin-binding protein utrophin (GF$\mathrm{P}:: \mathrm{utCH})$ in the sheath cells and mCherry::utCH in the

Figure 1 WASP functions in the glial cells to regulate ciliogenesis in C. elegans neurons. (A) Schematic of the C. elegans amphid. Top: two glial cells, including the socket cell (AMso, pink) and the sheath cell (AMsh, blue). Each amphid has 12 neurons (only one is drawn in brown). The area in the rectangle is enlarged in the bottom. (B) Schematic of the sensory compartment [3]. Neuronal cilia are extended from dendritic endings and enter the sensory channel that is formed by the glial cells. TZ: transition zone. (C) The morphology of the amphid (top) and phasmid (bottom) cilia labeled with OSM-6::mCherry in WT and wsp-1a (gm324) mutant animals. Yellow arrowheads: defects of the ciliary distal segments in wsp-1a. White arrowheads indicate the boundaries of the ciliary distal and middle segments. Scale bar, $2 \mu \mathrm{m}$. (D) Quantifications of cilium phenotypes in WT and wsp-1a mutant animals. $n=50-80$. (E) Kymographs (Middle (M) or Distal (D)) and corresponding lines (M' or D') of OSM-6::mCherry motility. Numbers represent mean \pm SD ( $n$ $=80-112$ ). Horizontal bar, $2 \mu \mathrm{m}$; vertical bar, $5 \mathrm{~s}$. (F) Cross-section TEM analysis of the amphid channel cilia in WT and wsp-1a. Representative images show the cross sections of the transition zone, middle segment, distal segment and distal tip. Yellow asterisks: highly electron-dense materials. Yellow arrowheads: collapse of the channel. Scale bar, $500 \mathrm{~nm}$. (G) Top (left) and side (right) views of a 3D reconstruction from the aligned cross-section FIB-SEM images show trajectories of cilia and the channel from the distal tip to the transition zone in WT and wsp-1a mutants. Colors: cilia. Grey: highly electron-dense material. Green: channel. Scale bar, $1 \mu \mathrm{m}$. (H) Longitudinal section TEM micrographs of the amphids in WT and wsp-1a mutant animals. Pink: socket cells. Blue: sheath cells. Brown: cilia. Yellow arrowheads: the collapsed socket cell. Yellow asterisks: highly electron-dense materials. Green asterisk: curved cilium. Scale bar, $1 \mu \mathrm{m}$. (I) Side views of a 3D reconstruction from the aligned longitudinal section FIB-SEM images show trajectories of cilia and the socket cells in WT and wsp-1a mutants. Colors: cilia. Pink: socket cells. Arrow: curved and branched cilia. Scale bar, 2 $\mu \mathrm{m}$. (J) Bottom views of a 3D reconstruction from the aligned longitudinal section FIB-SEM images show the socket cell morphology in WT and wsp-1a mutant animals. Scale bar, $2 \mu \mathrm{m}$. (K) 3D reconstruction image shows Y-shaped cilia in wsp-1a mutant animals. (L) The axonemal MT structure of the ciliary middle or distal segments. Red arrowhead: doublet MTs. Scale bar, $100 \mathrm{~nm}$. More images are shown in Supplementary information,Figure S1C. (M) Schematics of the axonemal MTs in WT and wsp-1a mutant cilia. (N-O) Localization of endogenous WSP-1A (N) and ARX-2 (O) around the amphid and phasmid of GFP knock-in strains. Cilia were labeled with OSM-6::mCherry (red). Arrowheads indicate the boundaries of the ciliary distal and middle segments. (P) Quantifications of the Dyf defect in the wsp-1a mutant and rescued animals. Two independent transgenic lines (L1 and L2) that express wsp-1a in the socket cells rescued Dyf defect in wsp-1a mutants. Error bars indicate SD $(n=350-400)$. (Q) Left: schematic of the phasmid socket cells (PHso1 and PHso2). Right: fluorescence images of F-actin (mCherry::utCH) in the phasmid socket cells of WT and wsp-1a mutant animals. (R) Quantifications of the length of F-actin fluorescence distribution in Q. Error bars indicate SD $(n=20-31)$. Scale bars in $\mathbf{N}, \mathbf{O}$, and $\mathbf{Q}, 2 \mu \mathrm{m}$. ${ }^{* *} P<0.01,{ }^{* * *} P<0.001$ based on Student's $t$-test; n.s., not significant. 

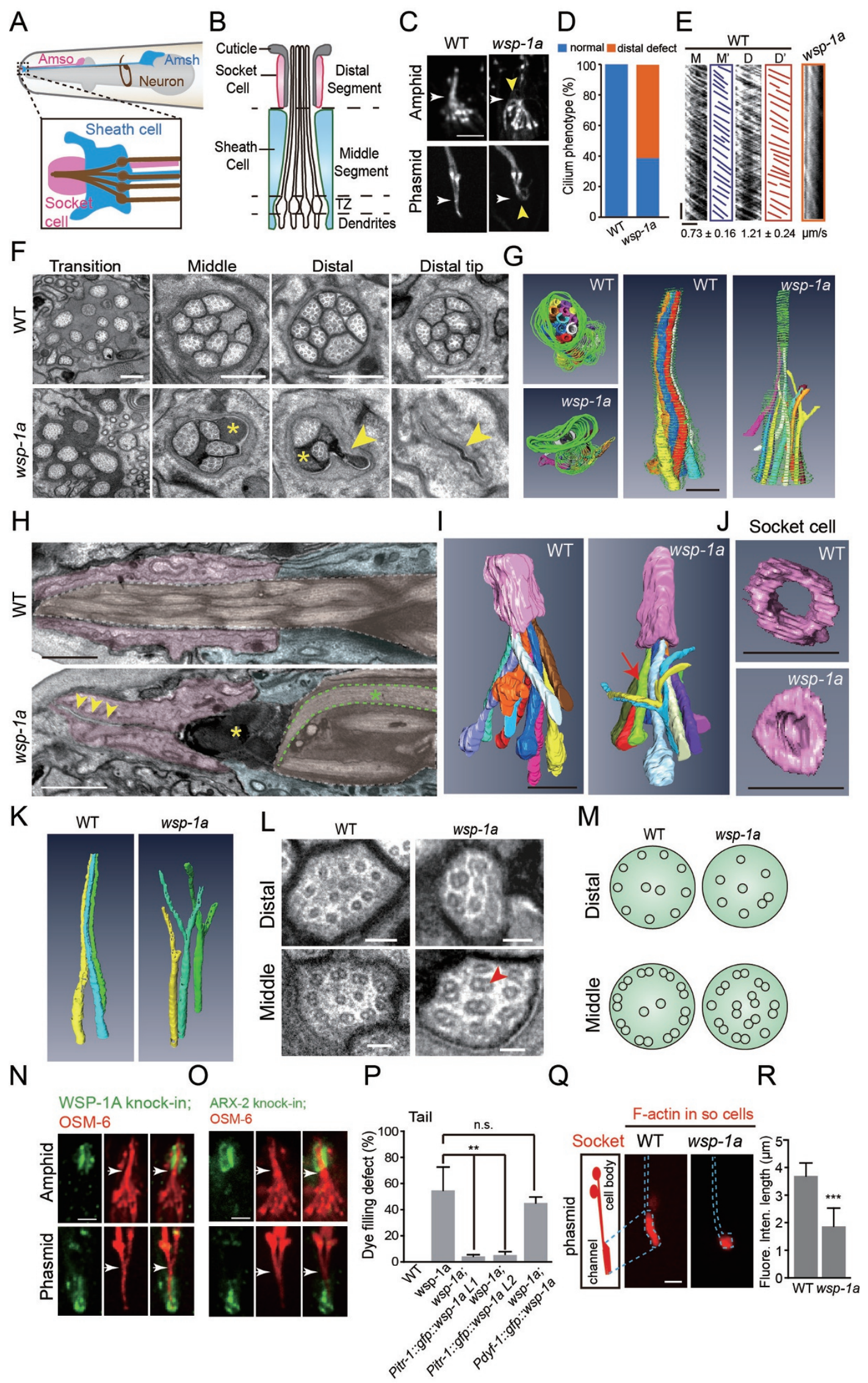
socket cells, we observed GFP and mCherry fluorescence inside the sheath and socket cells, respectively (Supplementary information, Figure S2C), indicative of actin filaments in these cells. In contrast, GFP::utCH expressed in ciliated neurons did not stain cilia with GFP fluorescence but accumulated at the dendritic ending where the transition zone locates (Supplementary information, Figure S2D), which is consistent with the notion that F-actin does not assemble inside cilia but may operate around the transition zone to regulate IFT-cargo recruitment [6, 9]. We further examined how the glial actin cytoskeleton is affected in animals lacking WASP function. While the mCherry::utCH fluorescence spanned approximately $4 \mu \mathrm{m}$ in the WT socket cells, mCherry::utCH was restricted at the distal tip of the socket cells in wsp-la mutants (Figure 1Q-1R). Consistently, ARX-2::GFP fluorescence was weakened in the wsp-1a-deficient socket cells compared with the WT cells $(n=10$; Supplementary information, Figure S2G).These results indicate that WASP regulates the size of sensory channel by modulating the organization of the glial actin cytoskeleton. WASP-Arp2/3-dependent actin polymerization is involved in the myelination by Schwann cells in the vertebrate peripheral nervous system [10], and the C. elegans WASP may similarly regulate the glial membrane extension to ensheath neuronal cilia.

In conclusion, our study reveals a new function of the actin cytoskeleton in metazoan ciliogenesis and highlights the importance of compartment-cilium interactions in this process. The reduction of channel size in wspla mutants disrupts the morphology of cilia inside the channel and the organization of axonemal MTs (Figure $1 \mathrm{~F}-1 \mathrm{M})$, suggesting that sensory compartment is not only crucial for the exposure of sensory cilia to the environment but also provides a "ciliary niche" for regulating cilium morphology and axonemal structure. The potential niche factors include physical support from the sensory channel and possibly molecules that are secreted from the glial cells. In support of the ciliary niche hypothesis, the secretory vesicular structures have been observed in the socket and sheath cells $[8,11]$, and sensory cilia can take up materials from the extracellular medium [3]. Conversely, the mutation of an IFT-kinesin OSM-3 within sensory cilia causes an accumulation of secretory vesicles containing electron-dense matrix material in the surrounding sheath cells [3], which may suggest non-cell autonomous functions for IFT-motors in the secretory pathway leading to functional interactions between the cilia and the surrounding glial cells. Importantly, glial ensheathment of neuronal cilia is prevalent in Drosophila sensory organ, mammalian olfactory epithelium and other tissues [8, 11, 12], and WASP is an evolutionarily conserved actin regulator, which suggest that our results may represent a general paradigm for cilium assembly and the organization of sensory organs.

\section{Acknowledgments}

GO thanks Drs D Huangfu, G Jansen and M Bettencourt Dias for their discussions regarding this project. We also thank the staff members L Xiaomin of Tsinghua University Branch of China National Center for Protein Sciences (Beijing, China) for providing the facility support. This work was supported by the National Basic Research Program of China (973 Program; 2013CB945600 and 2012CB945002), and the National Natural Science Foundation of China (31201048, 31222035, 31101002, 31100972, 31171295, 31190063 and 31501131).

Hao Zhu ${ }^{1}$, Lianwan Chen ${ }^{2}$, Yihong Yang ${ }^{1}$, Zhiwen Zhu', Xianliang Zhang ${ }^{1}$, Wei Li ${ }^{1}$, Long Miao', Yan Zhang ${ }^{1}$, Guangshuo $\mathrm{Ou}^{1}$

${ }^{1}$ Tsinghua-Peking Center for Life Sciences, School of Life Sciences, Tsinghua University, Beijing 100084, China; ${ }^{2}$ Institute of Biophysics, Chinese Academy of Sciences, Beijing 100081, China

Correspondence:Guangshuo $\mathrm{Ou}^{\mathrm{a}}$, Yan Zhang ${ }^{\mathrm{b}}$

${ }^{a}$ E-mail: guangshuoou@tsinghua.edu.cn

${ }^{b}$ E-mail: zhangyan1011@gmail.com

\section{Reference}

1 Ishikawa H, Marshall WF. Nat Rev Mol Cell Biol 2011; 12:222-234.

2 Doroquez DB, Berciu C, Anderson JR, et al. Elife 2014; 3:e1948.

3 Perkins LA, Hedgecock EM, Thomson JN, et al. Dev Biol 1986; 117:456-487.

4 Scholey JM. Annu Rev Cell Dev Biol 2013; 29:443-469.

5 Avasthi P, Onishi M, Karpiak J, et al. Curr Biol 2014; 24:2025-2032.

6 Cao J, Shen Y, Zhu L, et al. Nat Cell Biol 2012; 14:697-706.

7 Kim J, Lee JE, Heynen-Genel S, et al. Nature 2010; 464:1048-1051.

8 Oikonomou G, Perens EA, Lu Y, et al. PLoS Biol 2011; 9:e1001121.

9 Quarmby L. Curr Biol 2014; 24:R796-R798.

10 Nave K-A, Werner HB. Annu Rev Cell Dev Biol 2014; 30:503-533.

11 Shaham S. Cold Spring Harb Perspect Biol 2015; 8:4.

12 Gillespie PG, Walker RG. Nature 2001; 413:194-202.

(Supplementary information is linked to the online version of the paper on the Cell Research website.) 